\title{
A family of iterative methods that uses divided differences of first and second orders
}

\author{
J. A. Ezquerro ${ }^{a}$, M. Grau-Sánchez $^{\mathrm{b}}$, M. A. Hernández-Verón ${ }^{\mathrm{a}}$, M. Noguera $^{\mathrm{b}}$ \\ ${ }^{a}$ Dpt. of Mathematics and Computation, University of La Rioja \\ 26004 Logroño, Spain \\ E-mail address: <jezquer><mahernan>@unirioja.es \\ b Dpt. of Applied Mathematics II, Technical University of Catalonia \\ 08034 Barcelona, Spain \\ E-mail address: <miquel.grau $><$ miquel.noguera $>$ @upc.edu
}

\begin{abstract}
The family of fourth-order Steffensen-type methods proposed by Zheng, Li and Huang (Appl. Math. Comput. 217 (2011) 9592-9597) is extended to solve systems of nonlinear equations. This extension uses multidimensional divided differences of first and second orders. For a certain computational efficiency index, two optimal methods are identified in the family. Semilocal convergence is shown for one of these optimal methods under mild conditions. Moreover, a numerical example is given to illustrate the theoretical results.
\end{abstract}

Keywords: nonlinear equations, iterative methods, divided difference, order of convergence, efficiency.

2000 Mathematics Subject Classification: 65H10, 65Y20.

This work was supported in part by the project MTM2011-28636-C02-01 of the Spanish Ministry of Science and Innovation.

\section{Introduction}

The main goal of this paper consists of presenting some derivative-free iterative methods which use divided differences of first and second orders in $\mathbb{R}^{m}$. In this study, we are concerned with the local and semilocal convergence and the computational efficiency of a family of two-step iterative methods with order of convergence at least four for solving 
systems of nonlinear equations in $\mathbb{R}^{m}$. For this, we present an original procedure to obtain the equation of the local error, which is based on Taylor's series and considers divided differences of second order.

Firstly, we consider the scalar case before presenting some generalizations. This study is based on the parametric families of iterative methods given in [16, 22]. Another interesting work in the scalar case can be found in [12], where a variant of Steffensen's method of fourth-order convergence is presented.

So, let $f: I \subset \mathbb{R} \longrightarrow \mathbb{R}$ and $f(x)=0$, where $I$ is a neighborhood of a simple root $\alpha \in \mathbb{R}$. We consider the parametric family of iterative methods given by Zheng, Li and Huang [22] and defined by

$$
\left\{\begin{array}{l}
x_{0} \in I, \\
z_{n}=x_{n}+\nu f\left(x_{n}\right) \\
u_{n}=x_{n}-\frac{f\left(x_{n}\right)}{\left[x_{n}, z_{n} ; f\right]}, \\
x_{n+1}=u_{n}-\frac{f\left(u_{n}\right)}{\left[u_{n}, x_{n} ; f\right]+\left[u_{n}, x_{n}, z_{n} ; f\right]\left(u_{n}-x_{n}\right)}, \quad n \geq 0,
\end{array}\right.
$$

where $\nu \in \mathbb{R},[u, t ; f]=\frac{f(t)-f(u)}{t-u}$ and $[u, t, s ; f]=\frac{[t, s ; f]-[u, t ; f]}{s-u}$.

Next, we consider the system of nonlinear equations

$$
F(x)=0,
$$

where $F: D \subset \mathbb{R}^{m} \longrightarrow \mathbb{R}^{m}$ is a nonlinear differentiable function such that $F \equiv$ $\left(F_{1}, F_{2}, \ldots, F_{m}\right)$ with $F_{i}: D \subseteq \mathbb{R}^{m} \longrightarrow \mathbb{R}, 1 \leq i \leq m$, and $D$ is a non-empty open convex domain. We suppose that $F$ has a simple root $\alpha \in D$.

After that, a divided difference of first order of the function $F$ at the points $x, y$ of $D \subseteq \mathbb{R}^{m}(x \neq y)$ is defined by (see [5])

$$
[x, y ; F]=\int_{0}^{1} F^{\prime}(t x+(1-t) y) d t
$$

Analogously, from [5] a divided difference of second order of the function $F$ at the distinct points $x, y, z \in D$ is defined by

$$
[x, y, z ; F]=\int_{0}^{1} \int_{0}^{1-t} F^{\prime \prime}(t x+s y+(1-t-s) z) d s d t .
$$

Generalizations of fourth-order derivative-free Steffensen-type methods and their applications to $\mathbb{R}^{m}$ are also discussed in $[17,18,20]$. From now on, $M_{2}$ is used to denote the following Steffensen-type scheme:

$$
\left\{\begin{array}{l}
x_{0} \in D, \\
z_{n}=x_{n}+\nu F\left(x_{n}\right), \\
x_{n+1}=M_{2}\left(x_{n}\right)=x_{n}-\left[x_{n}, z_{n} ; F\right]^{-1} F\left(x_{n}\right), \quad n \geq 0,
\end{array}\right.
$$


where $\nu \in \mathbb{R}$.

Based on the previous Steffensen type scheme, Wang et al. [20] have generalized the method of Ren et al. [16] for scalar equations to systems of nonlinear equations, which is given by

$$
\left\{\begin{array}{l}
x_{0} \in D \\
z_{n}=x_{n}+\nu F\left(x_{n}\right), \\
u_{n}=M_{2}\left(x_{n}\right) \\
x_{n+1}=u_{n}-\left(\left[u_{n}, x_{n} ; F\right]+\left[u_{n}, z_{n} ; F\right]-\left[z_{n}, x_{n} ; F\right]\right)^{-1} F\left(u_{n}\right), \quad n \geq 0 .
\end{array}\right.
$$

They also extended the method given by Liu et al. [12] to solve systems of nonlinear equations in [20], which is given by

$$
\left\{\begin{array}{l}
x_{0} \in D, \\
z_{n}=x_{n}+\nu F\left(x_{n}\right), \\
u_{n}=M_{2}\left(x_{n}\right), \\
x_{n+1}=u_{n}-\left[u_{n}, x_{n} ; F\right]^{-1}\left(\left[u_{n}, x_{n} ; F\right]-\left[u_{n}, z_{n} ; F\right]+\left[z_{n}, x_{n} ; F\right]\right)\left[u_{n}, x_{n} ; F\right]^{-1} F\left(u_{n}\right), n \geq 0 .
\end{array}\right.
$$

Recently, based on (2), the following fourth-order derivative-free two-step method has been proposed by Sharma et al. in [18]:

$$
\left\{\begin{array}{l}
x_{0} \in D \\
z_{n}=x_{n}+\nu F\left(x_{n}\right), \\
u_{n}=M_{2}\left(x_{n}\right), \\
x_{n+1}=u_{n}-\left(3 I-\left[z_{n}, x_{n} ; F\right]^{-1}\left(\left[u_{n}, x_{n} ; F\right]+\left[u_{n}, z_{n} ; F\right]\right)\right)\left[z_{n}, x_{n} ; F\right]^{-1} F\left(u_{n}\right), n \geq 0 .
\end{array}\right.
$$

The main aim of this paper is to develop an efficient derivative-free family of fourthorder Steffensen-type methods as a modification of the method given in (1) and proposed by Zheng et al. in [22], so that we can use it in the multidimensional case. In a natural way, we can do it by writing the following parametric family of iterative methods:

$$
\left\{\begin{array}{l}
x_{0} \in D \\
z_{n}=x_{n}+\nu F\left(x_{n}\right), \\
u_{n}=M_{2}\left(x_{n}\right) \\
x_{n+1}=u_{n}-\left(\left[u_{n}, x_{n} ; F\right]+\left[x_{n}, z_{n}, u_{n} ; F\right]\left(u_{n}-x_{n}\right)\right)^{-1} F\left(u_{n}\right), \quad n \geq 0
\end{array}\right.
$$

where $\nu \in \mathbb{R}$.

In this work, we define a biparametric family of iterative methods, which includes (6) 
and a new parameter $\lambda \in \mathbb{R}$, in order to improve the efficiency, which is written as

$$
\left\{\begin{array}{l}
x_{0} \in D \\
y_{n}=x_{n}+\lambda F\left(x_{n}\right), \\
z_{n}=x_{n}+\nu F\left(x_{n}\right) \\
u_{n}=x_{n}-\left[y_{n}, z_{n} ; F\right]^{-1} F\left(x_{n}\right), \\
x_{n+1}=u_{n}-\left[\Phi\left(y_{n}, z_{n}, u_{n}\right)\right]^{-1} F\left(u_{n}\right), \quad n \geq 0
\end{array}\right.
$$

where $\lambda, \nu \in \mathbb{R}$, such that $\lambda$ and $\nu$ are not zero simultaneously, and

$$
\Phi\left(y_{n}, z_{n}, u_{n}\right)=\left[u_{n}, y_{n} ; F\right]+\left[y_{n}, z_{n}, u_{n} ; F\right]\left(u_{n}-y_{n}\right) .
$$

Observe that (7) is reduced to (6) if $\lambda=0$.

The paper is organized as follows. In Section 2, we obtain the inverse of the divided differences of first and second orders for the multidimensional case and we see that all the methods of family (7) have local order of convergence at least four. In Section 3, we analyse the computational efficiency of the methods and we identify two optimal representants of family (7). In Section 4, we study the semilocal convergence of one of these optimal methods by using a technique based on recurrence relations. Finally, in Section 5, we consider a numerical example where three of the most efficient methods of family (7) are applied to solve a system of nonlinear equations that arises from the discretization of a nonlinear integral equation of Hammerstein type.

\section{Local convergence}

From the well-known Genochi-Hermite formula (see $[5,9,15])$ we have that, if $x, x+$ $h, x+k \in D$ are distinct points of $\mathbb{R}^{m}$, the divided difference of first order of the function $F$ at the points $x, x+h$ can be defined by

$$
[x+h, x ; F]=\int_{0}^{1} F^{\prime}(x+t h) d t .
$$

Analogously, we define the divided difference of second order of $F$ at the points $x, x+$ $k, x+h$ by

$$
[x+h, x+k, x ; F]=\int_{0}^{1} \int_{0}^{1-t} F^{\prime \prime}(x+t h+s k) d s d t .
$$

From the previous expressions, if $F$ a is sufficiently differentiable function, we can obtain Taylor's series of the divided differences of first and second orders by developing $F^{\prime}$ and $F^{\prime \prime}$ in Taylor's series. Moreover, from the last, we can also obtain Taylor's series of their inverses. In consequence, we will be able to establish the local error equation. 


\subsection{A development of the inverse of the divided differences of first and second orders}

To obtain the vectorial error equation of the divided difference operators, we need the well-known Taylor theorem that is now included.

Theorem 2.1 If $F$ has continuous derivatives up to order $k+1$ in a convex open set $D \subset \mathbb{R}$ and $\left\|F^{k+1}(x)\right\| \leq \chi$, for all $x \in D$, then, if $x_{0} \in D$,

$$
F(x)=\sum_{j=0}^{k} \frac{1}{j !} F^{(j)}\left(x_{0}\right)\left(x-x_{0}\right)^{k}+R_{x_{0}, k}\left(x-x_{0}\right),
$$

where the remainder can be expressed as

$$
R_{x_{0}, k}\left(x-x_{0}\right)=\frac{1}{k !} \int_{0}^{1}(1-t)^{k} F^{(k+1)}\left(x_{0}+t\left(x-x_{0}\right)\right)\left(x-x_{0}\right)^{k+1} d t
$$

with

$$
\left\|R_{x_{0}, k}\left(x-x_{0}\right)\right\| \leq \frac{\chi}{(k+1) !}\left\|x-x_{0}\right\|^{k+1},
$$

and $R_{x_{0}, k}\left(x-x_{0}\right)=O\left(\left(x-x_{0}\right)^{k+1}\right)$.

By developing $F^{\prime}(x+t h)$ and $F^{\prime \prime}(x+t h+s k)$ in Taylor's series at the point $x$ and integrating, we obtain, from (9) and (10), respectively:

$$
\begin{aligned}
{[x+h, x ; F] } & =F^{\prime}(x)+\frac{1}{2} F^{\prime \prime}(x) h+O\left(h^{2}\right), \\
{[x+h, x+k, x ; F] } & =\frac{1}{2} F^{\prime \prime}(x)+\frac{1}{6} F^{\prime \prime \prime}(x)(h+k)+O_{2}(h, k),
\end{aligned}
$$

where $O\left(h^{2}\right)=\frac{1}{6} F^{\prime \prime \prime}(c) h^{2}$, the point $c$ belongs to the straight line between $x$ and $x+h$, and $h^{j}$ is $(h, \stackrel{j}{\cdot}, h) \in \mathbb{R}^{m} \times \stackrel{j}{\cdot} \times \mathbb{R}^{m}$. A function depending on $h$ and $k$ is denoted by $O_{p}(h, k)$ if it is $O\left(h^{q_{0}} k^{q_{1}}\right)$ with $q_{0}+q_{1}=p, q_{k} \geq 0, k=0,1$. Setting $e=x-\alpha$, we develop $F(x)$ and its derivatives in a neighborhood of $\alpha$ and, assuming that $\left[F^{\prime}(\alpha)\right]^{-1}$ exists, we have

$$
F(x)=\Gamma\left(e+A_{2} e^{2}+A_{3} e^{3}+O\left(e^{4}\right)\right),
$$

with $\Gamma=F^{\prime}(\alpha) \in \mathcal{L}\left(\mathbb{R}^{m}, \mathbb{R}^{m}\right), A_{j}=\frac{1}{j !} \Gamma^{-1} F^{(j)}(\alpha) \in \mathcal{L}_{j}\left(\mathbb{R}^{m}, \mathbb{R}^{m}\right), \quad j=2,3$, where $\mathcal{L}\left(\mathbb{R}^{m}, \mathbb{R}^{m}\right)$ and $\mathcal{L}_{j}\left(\mathbb{R}^{m}, \mathbb{R}^{m}\right)$ are the spaces of bounded linear and $j$-linear symmetric functions, respectively. From (13), we have

$$
\begin{aligned}
& F^{\prime}(x)=\Gamma\left(I+\sum_{k=2}^{3} k A_{k} e^{k-1}+O\left(e^{3}\right)\right), \\
& F^{\prime \prime}(x)=\Gamma\left(2 A_{2}+6 A_{3} e+O\left(e^{2}\right)\right) \\
& F^{\prime \prime \prime}(x)=\Gamma\left(6 A_{3}+O(e)\right)
\end{aligned}
$$




\subsection{Main result}

From the previous results, we obtain the following theorem for the local error equation of the family of iterative methods defined in (7).

Theorem 2.2 Let $F: D \subset \mathbb{R}^{m} \longrightarrow \mathbb{R}^{m}$ be a nonlinear four times continuously Fréchet differentiable function in $D$, where $D$ is a non-empty open convex domain and $\alpha \in D$ a simple solution of $F(x)=0$. Then, the family of iterative methods defined in (7) has local order of convergence at least four and satisfies the following local error equation

$$
e_{n+1}=A_{2} \varepsilon_{u}^{2}-A_{3} \varepsilon_{y} \varepsilon_{z} \varepsilon_{u}+O\left(e_{n}^{5}\right),
$$

where $\varepsilon_{y}, \varepsilon_{z}$ and $\varepsilon_{u}$ are given in (15), (16) and (21), respectively.

Proof. Hereafter, in this proof, we remove the subindexes written in (7), so that $x=x_{n}, y=y_{n}, u=u_{n}, z=z_{n}$ and $x=x_{n+1}$. Setting $\varepsilon_{x}=e, \varepsilon_{y}=y-\alpha, \varepsilon_{z}=z-\alpha$ and, using (13), we have

$$
\begin{aligned}
& \varepsilon_{y}=e+\lambda \tilde{e}+\lambda \Gamma A_{2} e^{2}+O\left(e^{3}\right), \\
& \varepsilon_{z}=e+\nu \tilde{e}+\nu \Gamma A_{2} e^{2}+O\left(e^{3}\right)
\end{aligned}
$$

where $\tilde{e}=\Gamma e$.

Next replacing expressions (13) and (14) into (11), we obtain

$$
[x+h, x ; F]=\Gamma\left(I+A_{2}(2 e+h)+A_{3}\left(3 e^{2}+3 e h+h^{2}\right)+O_{3}(h, x)\right),
$$

and setting $y=x+h$ and $h=\varepsilon_{y}-e$ in (17) we have

$$
[y, x ; F]=\Gamma\left(I+A_{2}\left(\varepsilon_{y}+e\right)+A_{3}\left(\varepsilon_{y}^{2}+\varepsilon_{y} e+e^{2}\right)+\ldots\right) .
$$

From (18) we take

$$
[y, z ; F]=\Gamma\left(I+A_{2}\left(\varepsilon_{y}+\varepsilon_{z}\right)+A_{3}\left(\varepsilon_{y}^{2}+\varepsilon_{y} \varepsilon_{z}+\varepsilon_{z}^{2}\right)+O_{3}\left(\varepsilon_{y}, \varepsilon_{z}\right)\right),
$$

and, by developing in a formal series expansion of $\varepsilon_{y}$ and $\varepsilon_{z}$ the inverse of $[y, z ; F]$, we get

$$
\begin{aligned}
{[y, z ; F]^{-1} } & =\left(I-A_{2}\left(\varepsilon_{y}+\varepsilon_{z}\right)+O_{2}\left(\varepsilon_{y}, \varepsilon_{z}\right)\right) \Gamma^{-1} \\
& =\left(I-A_{2}(2 e+\sigma \tilde{e})+O\left(e^{2}\right)\right) \Gamma^{-1},
\end{aligned}
$$

where $\sigma=\lambda+\nu$. By subtracting $\alpha$ from both sides of the third step of (7) and taking into account (20), we have

$$
\begin{aligned}
\varepsilon_{u}=u-\alpha & =e-\left(I-A_{2}(2 e+\sigma \tilde{e})+O\left(e^{2}\right)\right)\left(e+A_{2} e^{2}+O\left(e^{3}\right)\right) \\
& =A_{2}(e+\sigma \tilde{e}) e+O\left(e^{3}\right) .
\end{aligned}
$$


So, in the first step of (7) we have quadratic convergence.

Setting now $h=z-u=\varepsilon_{z}-\varepsilon_{u}, k=y-u=\varepsilon_{y}-\varepsilon_{u}$, and replacing expressions (13)-(14) again into (12), we have

$$
\begin{aligned}
{[y, z, u ; F] } & =\Gamma\left(A_{2}+A_{3}\left(\varepsilon_{y}+\varepsilon_{z}+\varepsilon_{u}\right)+O_{2}\left(\varepsilon_{y}, \varepsilon_{z}, \varepsilon_{u}\right)\right) \\
{[y, z, u ; F](u-y) } & =\Gamma\left(A_{2}\left(\varepsilon_{u}-\varepsilon_{y}\right)-A_{3}\left(\varepsilon_{y}+\varepsilon_{z}\right) \varepsilon_{y}+O_{3}\left(\varepsilon_{y}, \varepsilon_{z}, \varepsilon_{u}\right)\right)
\end{aligned}
$$

where a function depending on $h, k$ and $j$ is denoted by $O_{p}(h, k, j)$ if it is $O\left(h^{q_{0}} k^{q_{1}} j^{q_{2}}\right)$ with $q_{0}+q_{1}+q_{2}=p, q_{i} \geq 0,0 \leq i \leq 2$.

Taking into account (19) for operator $[u, y ; F]$

$$
[u, y ; F]=\Gamma\left(I+A_{2}\left(\varepsilon_{u}+\varepsilon_{y}\right)+A_{3} \varepsilon_{y}^{2}+O\left(e^{3}\right)\right),
$$

and expression (22) we obtain

$$
\Phi(y, z, u)=[u, y ; F]+[y, z, u ; F](u-y)=\Gamma\left(I+2 A_{2} \varepsilon_{u}-A_{3} \varepsilon_{y} \varepsilon_{z}+O\left(e^{3}\right)\right),
$$

and

$$
\Phi(y, z, u)^{-1}=\left(I-2 A_{2} \varepsilon_{u}+A_{3} \varepsilon_{y} \varepsilon_{z}+O\left(e^{3}\right)\right) \Gamma^{-1} .
$$

Subtracting $\alpha$ from both sides of the fourth step of (7), we obtain the following error equation for $(7)$

$$
\begin{aligned}
e_{n+1} & =\varepsilon_{u}-\left(I-2 A_{2} \varepsilon_{u}+A_{3} \varepsilon_{y} \varepsilon_{z}+O\left(e^{3}\right)\right)\left(\varepsilon_{u}+A_{2} \varepsilon_{u}^{2}+O\left(e^{6}\right)\right) \\
& =A_{2} \varepsilon_{u}^{2}-A_{3} \varepsilon_{y} \varepsilon_{z} \varepsilon_{u}+O\left(e^{5}\right) .
\end{aligned}
$$

Taking into consideration (15), (16) and (21), the last expression (24) proves that (7) has local order of convergence at least four.

\section{Efficiency analysis}

To analyze the efficiency of (7), we define the computational efficiency index $(C E I)$ by the order of convergence $\rho$ to the inverse power of the computational cost $\mathcal{C}$ (see $[9,15])$. In this work, $C E I=\rho^{1 / \mathcal{C}}$ with $\rho=4$ for all the methods. We define $\mathcal{C}=A(m) \mu+P(m)$, where $A(m)$ is the number of scalar evaluations per iteration, $\mu$ is the ratio between products and evaluations of functions that are required to express $\mathcal{C}$ in terms of products and $P(m)$ is the number of products of the algorithm per iteration.

We will consider the first order divided difference of defined by

$$
[\bar{u}, \bar{v} ; F]_{i j}=\frac{1}{u_{j}-v_{j}}\left(F_{i}\left(u_{1}, \ldots, u_{j}, v_{j+1}, \ldots, v_{m}\right)-F_{i}\left(u_{1}, \ldots, u_{j-1}, v_{j}, \ldots, v_{m}\right)\right),
$$


where $\bar{u} \in \mathbb{R}^{m}$ and $\bar{v} \in \mathbb{R}^{m}$ (see [15]). Observe that (9) is satisfied by the definition of divided difference (25) if the condition $[\bar{u}, \bar{v} ; F]=2[\bar{u}, 2 \bar{v}-\bar{u} ; F]-[\bar{v}, 2 \bar{v}-\bar{u} ; F]$, for all $(\bar{u}, \bar{v}) \in D \times D$ with $\bar{u} \neq \bar{v}$ and $2 \bar{v}-\bar{u} \in D$ is accomplished (for more details see $[14,15]$ ). In general, this is not true for any function $F$.

A development of Taylor of the expression considered in the previous numerical divided difference gives

$$
\begin{aligned}
\int_{0}^{1} D_{j} F_{i}(x+t h) d t & =D_{j} F_{i}(x)+\frac{1}{2} \sum_{k=1}^{m} D_{k j} F_{i}(x) h_{k}+\frac{1}{6} \sum_{k, \ell=1}^{m} D_{k \ell j} F_{i}(x) h_{k} h_{\ell}+O\left(h^{3}\right), \\
{[x+h, x ; F]_{i j} } & =D_{j} F_{i}(x)+\sum_{k=1}^{j-1} D_{k j} F_{i}(x) h_{k}+\frac{1}{2} D_{j j} F_{i}(x) h_{j}+O\left(h^{2}\right) .
\end{aligned}
$$

From the previous expansions we obtain

$$
\int_{0}^{1} D_{j} F_{i}(x+t h) d t-[x+h, x ; F]_{i j}^{(1)}=O(h) .
$$

Moreover, if we use the computational definition given in (25), then the expressions of errors (15) and (16) in the proof of Theorem 2.2 can be written as

$$
\begin{aligned}
& \varepsilon_{y}=e+\lambda \tilde{e}+\lambda \Gamma B_{2} e^{2}+O\left(e^{3}\right), \\
& \varepsilon_{z}=e+\nu \tilde{e}+\nu \Gamma B_{2} e^{2}+O\left(e^{3}\right),
\end{aligned}
$$

where $B_{2} \in \mathcal{L}_{2}\left(\mathbb{R}^{m}, \mathbb{R}^{m}\right)$. This fact can produce a decrease of convergence order in some algorithms. Now, the divided difference given in (19) is

$$
[y, z ; F]=\Gamma\left(I+C_{2}\left(\varepsilon_{y}+\varepsilon_{z}\right)+O_{2}\left(\varepsilon_{y}, \varepsilon_{z}\right)\right),
$$

where $C_{2} \in \mathcal{L}_{2}\left(\mathbb{R}^{m}, \mathbb{R}^{m}\right)$, and in general $C_{2} \neq A_{2}, B_{2}$. Furthermore, the expression of the error of $u$ given in (21) is

$$
\begin{aligned}
\varepsilon_{u}=u-\alpha & =e-\left(I-C_{2}\left(\varepsilon_{y}+\varepsilon_{z}\right)+O\left(e^{2}\right)\right)\left(e+A_{2} e^{2}+O\left(e^{3}\right)\right) \\
& =C_{2}(2 e+\sigma \tilde{e}) e-A_{2} e^{2}+O\left(e^{3}\right) .
\end{aligned}
$$

By using definition (25), the development of the operator $\Phi(y, z, u)$ given in $(23)$ is

$$
\Phi(y, z, u)=[u, y ; F]+[y, z, u ; F](u-y)=\Gamma\left(I+2 D_{2} \varepsilon_{u}-D_{3} \varepsilon_{y} \varepsilon_{z}+O\left(e^{3}\right)\right),
$$

where $D_{k} \in \mathcal{L}_{k}\left(\mathbb{R}^{m}, \mathbb{R}^{m}\right), k=2,3, D_{2} \neq A_{2}, B_{2}, C_{2}$ and $D_{3} \neq A_{3}$. Finally, we obtain the expression of $e_{n+1}$ (see (24))

$$
\begin{aligned}
e_{n+1} & =\varepsilon_{u}-\left(I-2 D_{2} \varepsilon_{u}+D_{3} \varepsilon_{y} \varepsilon_{z}+O\left(e^{3}\right)\right)\left(\varepsilon_{u}+A_{2} \varepsilon_{u}^{2}+O\left(e^{6}\right)\right) \\
& =\left(2 D_{2}-A_{2}\right) \varepsilon_{u}^{2}-D_{3} \varepsilon_{y} \varepsilon_{z} \varepsilon_{u}+O\left(e^{5}\right) .
\end{aligned}
$$


Note that the last expression proves that (7) also has local order of convergence at least four, and, in this case,we observe that the only difference with the theoretical result appears in the terms of fourth degree in $e$.

Moreover, setting three points $\bar{u}, \bar{v}, \bar{w} \in \mathbb{R}^{m}$, for the second divided difference of $F$ at $\bar{u}, \bar{v}, \bar{w}$, we can consider the bilinear operators defined by (see [5]):

$[\bar{u}, \bar{v}, \bar{w} ; F]_{i j k}=\frac{1}{v_{k}-w_{k}}\left\{\left[\bar{u},\left(v_{1}, \ldots, v_{k}, w_{k+1}, \ldots, w_{m}\right)\right]_{i j}-\left[\bar{u},\left(v_{1}, \ldots, v_{k-1}, w_{k}, \ldots, w_{m}\right)\right]_{i j}\right\}$.

When we evaluate $F=\left(F_{1}, F_{2}, \ldots, F_{m}\right)$, we do $m$ functions and, if we compute a divided difference $[x+\lambda F(x), x+\nu F(x) ; F]$, where $\lambda, \nu \neq 0$, we evaluate $m(m+1)$ scalar functions. In general, we have two evaluations of $F, F(x)$ and $F(u)$, and three different evaluations of divided differences, $[y, z ; F]$ with $m(m+1)$ evaluations, and $[u, y ; F]$ and $[z, u ; F]$ with $m^{2}$ evaluations for each one. Consequently, $A(m)=3 m(m+1)$.

The minimum of evaluations arises for $\lambda$ or $\nu$ are equal to zero. Then, if we suppose $\lambda=0$, the divided difference $[x, z ; F]$ requires $m^{2}$ evaluations, $[u, x ; F]$ requires $m(m-1)$ evaluations and $[z, u ; F]$ requires $m(m-1)$ evaluations. In addition, we have $A(m)=3 m^{2}$.

Moreover, we must add $m^{2}$ quotients for any divided difference. In order to compute an inverse, we have $m(m-1)(2 m-1) / 6$ products and $m(m-1) / 2$ quotients in the decomposition $L U$ and $m(m-1)$ products and $m$ quotients in the resolution of two triangular linear systems. If we consider that the parameters $\lambda$ and $\nu$ are integers, since the computational cost of $\lambda F(x)$ and $\nu F(x)$ is negligible, we compute two inverses and three divided differences, so that we have $P(m)=\frac{m}{3}\left(2 m^{2}+3 m-5\right)+\ell m(4 m+1)$, where we suppose that a quotient is equivalent to $\ell$ products.

So, in this work, we restrict our analysis to the following three cases:
1) $\lambda=0$ and $\nu=1$,
2) $\lambda=-1$ and $\nu=0$,
3) $\lambda=-1$ and $\nu=1$.

In the two first cases we have $\mathcal{C}(m)=3 m^{2} \mu+P(m)$ and, in the last case, we have $\mathcal{C}(m)=\left(3 m^{2}+3 m\right) \mu+P(m)$, so that 1$)$ and 2$)$ are the most efficient cases. Note that the value of $P(m)$ is the same in the three cases.

The computational efficiency of case 1) was studied also by Sharma and Arora in [17], where the iterative method is denoted by $M_{4,1}$ and compared with the new algorithm $M_{4,3}$. In a result of this paper it is proved that: if we denote by $\mathcal{C}_{4, j}, j=1,3$, the computational cost of the schemes $M_{4, j}, j=1,3$ respectively, then $\mathcal{C}_{4,1}(m) \leq \mathcal{C}_{4,3}(m)$ for $m \leq 4$ and independently of $\mu$.

\section{Semilocal convergence}

In this section, we study the semilocal convergence of an optimal method of the family of iterative methods defined in (7). Observe that, from the expression $([5,15])$

$$
[y, z, u ; F](u-y)=[z, u ; F]-[y, z ; F]
$$




$$
\Phi(y, z, u)=[u, y ; F]+[y, z, u ; F](u-y)=[u, y ; F]+[z, u ; F]-[y, z ; F],
$$

so that $(7)$ can be then written as

$$
\left\{\begin{array}{l}
x_{0} \in D \\
y_{n}=x_{n}+\lambda F\left(x_{n}\right) \\
z_{n}=x_{n}+\nu F\left(x_{n}\right) \\
u_{n}=x_{n}-\left[y_{n}, z_{n} ; F\right]^{-1} F\left(x_{n}\right), \\
x_{n+1}=u_{n}-\left(\left[u_{n}, y_{n} ; F\right]-\left[y_{n}, z_{n} ; F\right]+\left[z_{n}, u_{n} ; F\right]\right)^{-1} F\left(u_{n}\right), \quad n \geq 0 .
\end{array}\right.
$$

Notice that the last expression of algorithm (7) allows us to obtain a semilocal convergence result under mild convergence conditions.

Remember that the last algorithm is optimal when $\lambda=0$ and $\nu=1$ or when $\lambda=-1$ and $\nu=0$. In the following, we analyse the semilocal convergence of the optimal case given by $\lambda=0$ and $\nu=1$, which is the method $M_{4,1}$ described by Sharma and Arora ([17]) and in turn is a generalization of the method for scalar equations proposed by Ren, $\mathrm{Wu}$ and $\mathrm{Bi}$ in [16]. Note that the other optimal case is analogous to this (see the last algorithm). In addition, we consider the following iterative method (method (28) with $\lambda=0$ and $\nu=1)$ :

$$
\left\{\begin{array}{l}
x_{0} \in D \\
z_{n}=x_{n}+F\left(x_{n}\right) \\
u_{n}=x_{n}-A_{n}^{-1} F\left(x_{n}\right), \\
x_{n+1}=u_{n}-B_{n}^{-1} F\left(u_{n}\right), \quad n \geq 0
\end{array}\right.
$$

where

$$
\begin{aligned}
& A_{n}=\left[x_{n}, z_{n} ; F\right], \quad n \geq 0, \\
& B_{n}=\left[u_{n}, x_{n} ; F\right]-\left[x_{n}, z_{n} ; F\right]+\left[z_{n}, u_{n} ; F\right], \quad n \geq 0 .
\end{aligned}
$$

To prove the semilocal convergence of method (29), which has $R$-order of convergence at least four, we consider the classic convergence conditions that are required to analyse the semilocal convergence of classic iterative methods as the secant method, whose $R$-order of convergence is at least superlinear $([3,4])$, or Steffensen's method, whose $R$-order of convergence is at least two $([1,2,6])$. Consequently, the semilocal convergence conditions required in this work to method (29) are mild.

Firstly, we give the well-known Banach lemma on invertible operators [19].

Lemma 4.1 Let $L$ be a bounded linear function in $\mathbb{R}^{m}$ such that $\|L\| \leq \gamma<1$, then $I-A$ is invertible and $\left\|(I-L)^{-1}\right\| \leq \frac{1}{1-\gamma}$.

Secondly, we give the following technical lemma that we use later. The proof is immediate from (29) and the definition of the first-order divided difference. 
Lemma 4.2 Let $\left\{x_{n}\right\}$ be the sequence given in (29). If $u_{m-1} \neq x_{m}$ and $u_{m} \neq x_{m}$ with $z_{m-1}, u_{m-1}, x_{m}, u_{m} \in D$, then

$$
\begin{aligned}
F\left(u_{m}\right)= & \left(\left[u_{m}, x_{m} ; F\right]-A_{m}\right)\left(u_{m}-x_{m}\right), \quad m \geq 0, \\
F\left(x_{m}\right)= & \left(\left[x_{m}, u_{m-1} ; F\right]-\left[u_{m-1}, x_{m-1} ; F\right]\right. \\
& \left.+\left[x_{m-1}, z_{m-1} ; F\right]-\left[z_{m-1}, u_{m-1} ; F\right]\right)\left(x_{m}-u_{m-1}\right), \quad m \geq 1 .
\end{aligned}
$$

Thirdly, we suppose that there exists a first-order divided difference $[\cdot, \cdot ; F] \in \mathcal{L}\left(\mathbb{R}^{m}, \mathbb{R}^{m}\right)$ and

(C1) $\left\|F\left(x_{0}\right)\right\|=\delta_{0}$,

(C2) the divided difference $A_{0}=\left[x_{0}, z_{0} ; F\right]$ is invertible and such that $\left\|A_{0}^{-1}\right\| \leq \beta$,

$(C 3)\|[x, y ; F]-[u, v ; F]\| \leq K(\|x-u\|+\|y-v\|), K \geq 0, x, y, u, v \in D, x \neq y, u \neq v$.

Fourthly, a system of recurrence relations is provided in the next lemma, so that we can guarantee that sequence (29) is well-defined from it.

Lemma 4.3 Let $g$ and $h$ be the scalar functions defined by

$$
g(t)=\tilde{\beta}(1+K \hat{\beta}(1+\tilde{\beta}) t), \quad h(t)=2+K \hat{\beta} \tilde{\beta} t
$$

If conditions $(C 1)-(C 3)$,

$$
\begin{gathered}
M \equiv K \beta\left(2 R+\delta_{0}\right)<1, \\
N \equiv(3+2 \tilde{\beta}) K \tilde{\beta} \delta_{0}<1, \\
\delta_{0} \sqrt{P h\left(\delta_{0}\right)}<1
\end{gathered}
$$

are satisfied and there exists $R>0$ such that $z_{n}, u_{n}, x_{n+1} \in B\left(x_{0}, R\right) \subset D$, for all $n \geq 0$, then the following items are true for all $n \geq 1$ :

[I] $\left\|F\left(x_{n}\right)\right\| \leq \delta_{n}$

[II] there exists $A_{n}^{-1}$ and $\left\|A_{n}^{-1}\right\| \leq \tilde{\beta}$,

[III] $\delta_{n}<\delta_{n-1}$,

[IV] there exists $B_{n}^{-1}$ and $\left\|B_{n}^{-1}\right\| \leq \hat{\beta}$,

[V] $\left\|z_{n}-x_{n}\right\| \leq \delta_{n}$

[VI] $\left\|z_{n}-x_{0}\right\| \leq \delta_{n}+\sum_{i=0}^{n-1} g\left(\delta_{i}\right) \delta_{i}$,

[VII] $\left\|u_{n}-x_{n}\right\| \leq \tilde{\beta} \delta_{n}$, 


$$
\begin{gathered}
{[\boldsymbol{V I I I}]\left\|u_{n}-x_{0}\right\| \leq \tilde{\beta} \delta_{n}+\sum_{i=0}^{n-1} g\left(\delta_{i}\right) \delta_{i},} \\
{[\boldsymbol{I} \boldsymbol{X}]\left\|u_{n}-z_{n}\right\| \leq(1+\tilde{\beta}) \delta_{n},} \\
{[\boldsymbol{X}]\left\|F\left(u_{n}\right)\right\| \leq K \tilde{\beta}(1+\tilde{\beta}) \delta_{n}^{2}} \\
{[\boldsymbol{X I}]\left\|x_{n+1}-x_{n}\right\| \leq g\left(\delta_{n}\right) \delta_{n}} \\
{[\boldsymbol{X I I}]\left\|x_{n+1}-x_{0}\right\| \leq \sum_{i=0}^{n} g\left(\delta_{i}\right) \delta_{i}}
\end{gathered}
$$

Proof. From the last conditions, if $N_{0}=(3+2 \beta) K \beta \delta_{0}<1$, it follows that $B_{0}^{-1}$ exists and is such that $\left\|B_{0}^{-1}\right\| \leq \hat{\beta}_{0}$, where $\hat{\beta}_{0}=\frac{\beta}{1-N_{0}}$, since

$$
\left\|I-A_{0}^{-1} B_{0}\right\| \leq\left\|A_{0}^{-1}\right\|\left\|A_{0}-B_{0}\right\| \leq N_{0} .
$$

In addition,

$$
\begin{aligned}
\left\|z_{0}-x_{0}\right\| & =\left\|F\left(x_{0}\right)\right\| \leq \delta_{0}, \\
\left\|u_{0}-x_{0}\right\| & \leq\left\|A_{0}^{-1}\right\|\left\|F\left(x_{0}\right)\right\| \leq \beta \delta_{0}, \\
\left\|F\left(u_{0}\right)\right\| & \leq\left\|\left[u_{0}, x_{0} ; F\right]-A_{0}\right\|\left\|u_{0}-x_{0}\right\| \leq K \beta(1+\beta) \delta_{0}^{2}, \\
\left\|x_{1}-x_{0}\right\| & \leq\left\|u_{0}-x_{0}\right\|+\left\|B_{0}^{-1}\right\|\left\|F\left(u_{0}\right)\right\| \leq \beta\left(1+K \hat{\beta}_{0}(1+\beta) \delta_{0}\right) \delta_{0} .
\end{aligned}
$$

After that, we suppose that there exists $R>0$ such that $z_{0}, u_{0}, x_{1} \in B\left(x_{0}, R\right) \subset D$. Then,

$$
\begin{aligned}
\left\|F\left(x_{1}\right)\right\| & \leq\left(\left\|\left[x_{1}, u_{0} ; F\right]-\left[u_{0}, x_{0} ; F\right]\right\|+\left\|\left[x_{0}, z_{0} ; F\right]-\left[z_{0}, u_{0} ; F\right]\right\|\right)\left\|x_{1}-u_{0}\right\|, \\
& \leq K\left(\left\|B_{0}^{-1}\right\|\left\|F\left(u_{0}\right)\right\|+2\left(1+\left\|A_{0}^{-1}\right\|\right)\left\|F\left(x_{0}\right)\right\|\right)\left\|B_{0}^{-1}\right\| F\left(u_{0}\right) \| \\
& \leq K^{2} \hat{\beta}_{0} \beta(1+\beta)^{2}\left(2+K \hat{\beta}_{0} \beta \delta_{0}\right) \delta_{0}^{3} \\
& =\tilde{\delta}_{1} .
\end{aligned}
$$

We now observe that $\tilde{\delta}_{1}<\delta_{0}$, provided that

$$
K^{2} \hat{\beta}_{0} \beta(1+\beta)^{2}\left(2+K \hat{\beta}_{0} \beta \delta_{0}\right) \delta_{0}^{2}<1 .
$$

Moreover, from (31), it follows that the operator $A_{1}^{-1}$ exists and is such that $\left\|A_{1}^{-1}\right\| \leq \tilde{\beta}$, where $\tilde{\beta}=\frac{\beta}{1-M}$, since

$$
\left\|I-A_{0}^{-1} A_{1}\right\| \leq\left\|A_{0}^{-1}\right\|\left\|A_{0}-A_{1}\right\| \leq M .
$$

Therefore, $u_{1}$ is well-defined. 
Furthermore, taking into account (32) and (34), we have that $B_{1}^{-1}$ exists and is such that $\left\|B_{1}^{-1}\right\| \leq \hat{\beta}$, where $\hat{\beta}=\frac{\tilde{\beta}}{1-N}$, since

$$
\left\|I-A_{1}^{-1} B_{1}\right\| \leq\left\|A_{1}^{-1}\right\|\left\|A_{1}-B_{1}\right\| \leq N
$$

Therefore, $x_{2}$ is well-defined.

Notice that $\tilde{\beta}$ and $\hat{\beta}$ depend on $R$.

Now, we write

$$
\left\|x_{1}-x_{0}\right\| \leq g\left(\delta_{0}\right) \delta_{0}
$$

since $\beta<\tilde{\beta}, N_{0}<N$ and $\hat{\beta}_{0}<\hat{\beta}$.

Next, if we denote $\delta_{1}=P h\left(\delta_{0}\right) \delta_{0}^{3}$, where $P=K^{2} \hat{\beta} \tilde{\beta}(1+\tilde{\beta})^{2}$, we have $\tilde{\delta}_{1}<\delta_{1}$, so that we can write $\left\|F\left(x_{1}\right)\right\|<\delta_{1}$. In addition, if we suppose

$$
\operatorname{Ph}\left(\delta_{0}\right) \delta_{0}^{2}<1,
$$

it is clear that $\delta_{1}<\delta_{0}$ and (34) holds.

On the other hand, if (35) is satisfied, then (34) also is and consequently

$$
\begin{aligned}
\left\|z_{1}-x_{1}\right\| & =\left\|F\left(x_{1}\right)\right\| \leq \tilde{\delta}_{1}<\delta_{1}, \\
\left\|z_{1}-x_{0}\right\| & =\left\|z_{1}-x_{1}\right\|+\left\|x_{1}-x_{0}\right\|<\delta_{0}+g\left(\delta_{0}\right) \delta_{0}, \\
\left\|u_{1}-x_{1}\right\| & \leq\left\|A_{1}^{-1}\right\|\left\|F\left(x_{1}\right)\right\| \leq \tilde{\beta} \tilde{\delta}_{1}<\tilde{\beta} \delta_{1}, \\
\left\|u_{1}-x_{0}\right\| & \leq\left\|u_{1}-x_{1}\right\|+\left\|x_{1}-x_{0}\right\|<\tilde{\beta} \delta_{0}+g\left(\delta_{0}\right) \delta_{0}, \\
\left\|u_{1}-z_{1}\right\| & \leq\left(1+\left\|A_{1}^{-1}\right\|\right)\left\|F\left(x_{1}\right)\right\| \leq(1+\tilde{\beta}) \delta_{1}, \\
\left\|F\left(u_{1}\right)\right\| & \leq\left\|\left[u_{1}, x_{1} ; F\right]-A_{1}\right\|\left\|u_{1}-x_{1}\right\|<K \tilde{\beta}(1+\tilde{\beta}) \delta_{1}^{2}, \\
\left\|x_{2}-x_{1}\right\| & \leq\left\|u_{1}-x_{1}\right\|+\left\|B_{1}^{-1}\right\|\left\|F\left(u_{1}\right)\right\| \leq g\left(\delta_{1}\right) \delta_{1}, \\
\left\|x_{2}-x_{0}\right\| & \leq\left\|x_{2}-x_{1}\right\|+\left\|x_{1}-x_{0}\right\| \leq g\left(\delta_{1}\right) \delta_{1}+g\left(\delta_{0}\right) \delta_{0} .
\end{aligned}
$$

We now define the following scalar sequence:

$$
\delta_{n+1}=\operatorname{Ph}\left(\delta_{n}\right) \delta_{n}^{3}, \quad n \geq 0,
$$

where $P=K^{2} \hat{\beta} \tilde{\beta}(1+\tilde{\beta})^{2}$. Observe that $\left\{\delta_{n}\right\}$ is a strictly decreasing sequence and satisfies

$$
\delta_{n} \leq \frac{\left(\delta_{0} \sqrt{P h\left(\delta_{0}\right)}\right)^{3^{n}}}{\sqrt{P h\left(\delta_{0}\right)}}, \quad n \geq 1,
$$

provided that condition (33) holds. Besides, (35) holds if (33) does.

The proof of the lemma is completed in a similar way that the above-mentioned and using induction hypotheses.

After that, we give a technical lemma that establishes the influence of the parameter $\tilde{\beta}$ in the semilocal convergence of method (29). The proof of the lemma is immediate. 
Lemma 4.4 Suppose that conditions $(C 1)-(C 3)$ and (31) are satisfied.

(i) If $\beta\left(1+K \delta_{0}\right)>1$, then $\tilde{\beta}>1$ for all $R>0$.

(ii) If $\beta\left(1+K \delta_{0}\right)<1$, then
(a) $\tilde{\beta}<1$ for all $R \in\left(0, \frac{1-\beta\left(1+K \delta_{0}\right)}{2 K \beta}\right)$,
(b) $\tilde{\beta}>1$ for all $R \in\left(\frac{1-\beta\left(1+K \delta_{0}\right)}{2 K \beta},+\infty\right)$.

Notice that we need that the sequences $\left\{z_{n}\right\},\left\{u_{n}\right\}$ and $\left\{x_{n}\right\}$ are in the domain $D$ in order to prove the semilocal convergence of method (29), so that the first-order divided difference, $F\left(u_{n}\right)$ and $F\left(x_{n}\right)$ can be defined. For this, we give the following result

Lemma 4.5 Let $g$ and $h$ be the scalar functions defined in (30). Suppose that conditions (C1)-(C3), (31), (32) and (33) are satisfied. If there exists $R>0$ such that $x_{n}, z_{n}, u_{n} \in B\left(x_{0}, R\right) \subset D$, for all $n \geq 0$, then the following items are true for all $n \geq 1$ :

(i) $g\left(\delta_{n}\right) \delta_{n} \leq Q\left(\delta_{0} \sqrt{\operatorname{Ph}\left(\delta_{0}\right)}\right)^{3^{n}}$,

(ii) $\left\|z_{n}-x_{0}\right\| \leq \delta_{0}+\frac{g\left(\delta_{0}\right) \delta_{0}}{1-\delta_{0} \sqrt{P h\left(\delta_{0}\right)}}$,

(iii) $\left\|u_{n}-x_{0}\right\| \leq \tilde{\beta} \delta_{0}+\frac{g\left(\delta_{0}\right) \delta_{0}}{1-\delta_{0} \sqrt{P h\left(\delta_{0}\right)}}$,

(iv) $\left\|x_{n+1}-x_{0}\right\| \leq \frac{g\left(\delta_{0}\right) \delta_{0}}{1-\delta_{0} \sqrt{P h\left(\delta_{0}\right)}}$,

where $Q=\frac{g\left(\delta_{0}\right)}{\sqrt{\operatorname{Ph}\left(\delta_{0}\right)}}$.

Proof. To prove (i), it is sufficient to note that the function $g$ is non-decreasing and the sequence $\left\{\delta_{n}\right\}$ is strictly decreasing and satisfies condition (36).

Item (ii) follows easily from (i), since

$$
\begin{gathered}
\left\|z_{n}-x_{0}\right\| \leq\left\|z_{n}-x_{n}\right\|+\sum_{i=0}^{n-1}\left\|x_{i+1}-x_{i}\right\| \leq \delta_{n}+\sum_{i=0}^{n-1} g\left(\delta_{i}\right) \delta_{i} \\
<\delta_{0}+Q \sum_{i=0}^{n-1}\left(\delta_{0} \sqrt{P h\left(\delta_{0}\right)}\right)^{3^{i}}<\delta_{0}+Q \sum_{i \geq 0}\left(\delta_{0} \sqrt{P h\left(\delta_{0}\right)}\right)^{i+1}<\delta_{0}+\frac{g\left(\delta_{0}\right) \delta_{0}}{1-\delta_{0} \sqrt{P h\left(\delta_{0}\right)}} .
\end{gathered}
$$


Item (iii) follows analogously to item (ii) and item (iv) is a consequence of items [XII] of Lemma 4.3 and (i).

As we can see in the above-mentioned, the inequalities $\tilde{\beta}>1$ or $\tilde{\beta}<1$ determine which the bounds for the distances $\left\|z_{n}-x_{0}\right\|$ and $\left\|u_{n}-x_{0}\right\|$ have to be established.

Before presenting the semilocal convergence result for method (29), we give the next auxiliary scalar functions:

$$
\begin{aligned}
& f_{1}(R)=1-M=1-K \beta\left(2 R+\delta_{0}\right), \\
& f_{2}(R)=1-N=1-(3+2 \tilde{\beta}) K \tilde{\beta} \delta_{0}, \\
& f_{3}(R)=1-\delta_{0} \sqrt{P h\left(\delta_{0}\right)}, \\
& f_{4}(R)=R-\tilde{\beta} \delta_{0}-\frac{g\left(\delta_{0}\right) \delta_{0}}{1-\delta_{0} \sqrt{P h\left(\delta_{0}\right)}}, \\
& f_{5}(R)=R-\delta_{0}-\frac{g\left(\delta_{0}\right) \delta_{0}}{1-\delta_{0} \sqrt{P h\left(\delta_{0}\right)}} .
\end{aligned}
$$

Observe that conditions (31), (32) and (33) are reduced to $f_{1}(R)>0, f_{2}(R)>0$ and $f_{3}(R)>0$, respectively.

Theorem 4.6 Let $F: D \subseteq \mathbb{R}^{m} \longrightarrow \mathbb{R}^{m}$ be a continuous nonlinear function defined on a non-empty open convex domain $D$. We suppose that there exists $[u, v ; F] \in \mathcal{L}(D, D)$, for all $u, v \in D(u \neq v)$, and conditions $(C 1)-(C 3)$ are satisfied.

(i) If $\beta\left(1+K \delta_{0}\right)>1$ and there exists $R>0$ such that $f_{1}(R)>0, f_{2}(R)>0, f_{3}(R)>0$, $f_{4}(R) \geq 0$ and $B\left(x_{0}, R\right) \subset D$, then the sequence $\left\{x_{n}\right\}$, defined in (29), converges to a solution $x^{*}$ of $F(x)=0$.

(ii) If $\beta\left(1+K \delta_{0}\right)<1$ and there exists $R \in\left(0, \frac{1-\beta\left(1+K \delta_{0}\right)}{2 K \beta}\right)$ such that $f_{1}(R)>0$, $f_{2}(R)>0, f_{3}(R)>0, f_{5}(R) \geq 0$ and $B\left(x_{0}, R\right) \subset D$, then the sequence $\left\{x_{n}\right\}$, defined in (29), converges to a solution $x^{*}$ of $F(x)=0$.

(iii) If $\beta\left(1+K \delta_{0}\right)<1$ and there exists $R \in\left(\frac{1-\beta\left(1+K \delta_{0}\right)}{2 K \beta},+\infty\right)$ such that $f_{1}(R)>0$, $f_{2}(R)>0, f_{3}(R)>0, f_{4}(R) \geq 0$ and $B\left(x_{0}, R\right) \subset D$, then the sequence $\left\{x_{n}\right\}$, defined in (29), converges to a solution $x^{*}$ of $F(x)=0$.

Moreover, the solution $x^{*}$ and the iterates $x_{n}$ belong to $\overline{B\left(x_{0}, R\right)}$. Furthermore, the solution $x^{*}$ is unique in $B\left(x_{0}, r\right) \cap D$, where $r=\frac{1}{K \beta}-\delta_{0}-R$, provided that $R<\frac{1}{K \beta}-\delta_{0}$.

Proof. The fact that the sequence $\left\{x_{n}\right\}$ is well-defined follows easily from Lemma 4.5. In addition, for $m, n \geq 1$, it follows from item (i) of Lemma 4.5 that

$$
\left\|x_{m+n}-x_{n}\right\| \leq \sum_{i=n+1}^{m+n}\left\|x_{i}-x_{i-1}\right\| \leq \sum_{i=n+1}^{m+n} g\left(\delta_{i-1}\right) \delta_{i-1}
$$




$$
\begin{gathered}
<Q \sum_{i=n+1}^{m+n}\left(\delta_{0} \sqrt{P h\left(\delta_{0}\right)}\right)^{3^{i-1}} \leq Q\left(\delta_{0} \sqrt{P h\left(\delta_{0}\right)}\right)^{n} \sum_{i=0}^{m-1}\left(\delta_{0} \sqrt{P h\left(\delta_{0}\right)}\right)^{i} \\
=Q\left(\delta_{0} \sqrt{P h\left(\delta_{0}\right)}\right)^{n} \frac{1-\left(\delta_{0} \sqrt{P h\left(\delta_{0}\right)}\right)^{m}}{1-\delta_{0} \sqrt{P h\left(\delta_{0}\right)}}
\end{gathered}
$$

and, consequently, $\left\{x_{n}\right\}$ is a Cauchy sequence, since $\delta_{0} \sqrt{P h\left(\delta_{0}\right)}<1$. Therefore, $\left\{x_{n}\right\}$ is convergent.

If $\lim _{n \rightarrow \infty} x_{n}=x^{*}$, then it is clear that $x^{*} \in \overline{B\left(x_{0}, R\right)}$. Besides, $x^{*}$ is a solution of $F(x)=0$, since

$$
\left\|F\left(x_{n}\right)\right\| \leq \delta_{n} \leq \frac{\left(\delta_{0} \sqrt{P h\left(\delta_{0}\right)}\right)^{3^{n}}}{\sqrt{\operatorname{Ph}\left(\delta_{0}\right)}}
$$

and $\left\|F\left(x_{n}\right)\right\| \rightarrow 0$ by letting $n \rightarrow \infty$. Consequently, by the continuity of $F$, we obtain $F\left(x^{*}\right)=0$.

Finally, we prove the uniqueness of the solution $x^{*}$ in $B\left(x_{0}, r\right) \cap D$. Suppose that $y^{*}$ is another solution of $F(x)=0$ in $B\left(x_{0}, r\right) \cap D\left(x^{*} \neq y^{*}\right)$. If $L=\left[y^{*}, x^{*} ; F\right]$ is invertible, it follows that $F(x)=0$, since $L\left(y^{*}-x^{*}\right)=F\left(y^{*}\right)-F\left(x^{*}\right)$. To see this, we use

$$
\left\|I-A_{0}^{-1} L\right\| \leq\left\|A_{0}^{-1}\right\|\left\|A_{0}-L\right\| \leq K \beta\left(\left\|y^{*}-x_{0}\right\|+\left\|x^{*}-z_{0}\right\|\right)<K \beta\left(r+R+\delta_{0}\right)=1
$$

and the Banach lemma on invertible operators.

\section{Application}

Now, we present an application of the above analysis, where a system of nonlinear equations that arises from a process of discretization of a nonlinear integral equation of mixed Hammerstein type [13] is considered.

So, we consider

$$
x(s)=1+\frac{1}{3} \int_{0}^{1} G(s, t) x(t)^{2} d t, \quad s \in[0,1],
$$

where $x \in C[0,1], t \in[0,1]$, and the kernel $G$ is the Green function

$$
G(s, t)= \begin{cases}(1-s) t, & t \leq s, \\ s(1-t), & s \leq t .\end{cases}
$$

Firstly, we write the integral equation as $\mathcal{F}(x)=0$, where $\mathcal{F}: C[0,1] \rightarrow C[0,1]$ and

$$
\mathcal{F}(x)(s)=x(s)-1-\frac{1}{3} \int_{0}^{1} G(s, t) x(t)^{2} d t, \quad s \in[0,1] .
$$


Then, we transform it into a finite dimensional problem by using a Gauss-Legendre quadrature formula with 8 nodes:

$$
\int_{0}^{1} \varphi(t) d t \approx \sum_{i=1}^{8} w_{i} \varphi\left(t_{i}\right)
$$

where the nodes $t_{i}$ and the weights $w_{i}$ are known.

Denoting the approximation of $x\left(t_{i}\right)$ by $x_{i}, 1 \leq i \leq 8$, we obtain the following nonlinear system:

$$
x_{i}-1-\frac{1}{3} \sum_{j=1}^{8} a_{i j} x_{j}^{2}=0, \quad \text { where } \quad a_{i j}=\left\{\begin{array}{l}
w_{j} t_{j}\left(1-t_{i}\right) \text { if } j \leq i, \\
w_{j} t_{i}\left(1-t_{j}\right) \text { if } j>i,
\end{array} \quad i=1,2, \ldots, 8 .\right.
$$

After that, system (37) is written in matrix form as

$$
F(\bar{x}) \equiv \bar{x}-\overline{1}-\frac{1}{3} A \hat{x}=0
$$

where $F: \mathbb{R}^{8} \longrightarrow \mathbb{R}^{8}, \bar{x}=\left(x_{1}, x_{2}, \ldots, x_{8}\right)^{T}, \overline{1}=(1,1, \ldots, 1)^{T}, A=\left(a_{i j}\right)_{i, j=1}^{8}$ and $\hat{x}=$ $\left(x_{1}^{2}, x_{2}^{2}, \ldots, x_{8}^{2}\right)^{T}$.

To apply Theorem 4.6, we consider the divided differences given in (25) and (26), that satisfy (27), as we have seen before, and have first to prove that condition $(C 3)$ is satisfied. For this, according to (25), we have that the divided difference of first order is

$$
[\bar{u}, \bar{v} ; F]=I-\frac{1}{3} A \operatorname{diag}\{\bar{u}+\bar{v}\}, \quad \text { for } \quad \bar{u}, \bar{v} \in \mathbb{R}^{8} .
$$

If we now choose the max-norm, we have

$$
\|[\bar{u}, \bar{v} ; F]-[\bar{w}, \bar{z} ; F]\|_{\infty} \leq \frac{1}{3}\|A\|_{\infty}\left(\|\bar{u}-\bar{w}\|_{\infty}+\|\bar{v}-\bar{z}\|_{\infty}\right),
$$

where $\bar{u}=\left(u_{1}, u_{2}, \ldots, u_{8}\right)^{T}, \bar{v}=\left(v_{1}, v_{2}, \ldots, v_{8}\right)^{T}, \bar{w}=\left(w_{1}, w_{2}, \ldots, w_{8}\right)^{T}, \bar{z}=\left(z_{1}, z_{2}, \ldots, z_{8}\right)^{T}$ and $\|A\|_{\infty}=0.1235 \ldots$, so that $K=0.0411 \ldots$ and $(C 3)$ holds.

Now, we choose the starting point $\bar{x}_{0}=(1,1, \ldots, 1)^{T}$ and obtain $\delta_{0}=0.0411 \ldots$ and $\beta=1.0886 \ldots$ for Theorem 4.6. Therefore, $\beta\left(1+K \delta_{0}\right)=1.0887 \ldots>1$, so that we are in the first case of Theorem 4.6. In addition, $R=0.0906 \ldots>0$ is such that $f_{i}(R)>0$, for $i=1,2,3,4$, and the hypotheses of Theorem 4.6 are satisfied, and consequently, method (29) converges to the solution $\bar{x}^{*}=\left(x_{1}^{*}, x_{2}^{*}, \ldots, x_{8}^{*}\right)^{T}$ given in Table 1.

Notice that the starting point is the same for the three methods tested. The classical stopping criteria $\left\|e_{I+1}\right\|=\left\|x_{I+1}-\alpha\right\|<\varepsilon$ and $\left\|e_{I}\right\|>\varepsilon$, with $\varepsilon=10^{-\kappa}$ and $\kappa=4096$, is replaced by $\left\|\breve{e}_{I+1}\right\|<10^{-\eta}$ and $\left\|\breve{e}_{I}\right\|>10^{-\eta}$, with $\eta=\left[\kappa(\rho-1) / \rho^{2}\right]$, and

$$
\breve{e}_{n}=\left(\frac{F_{r}\left(x_{n}\right)}{F_{r}\left(x_{n-1}\right)}\right)_{1 \leq r \leq m},
$$




\begin{tabular}{|c|c||c|c||c|c||c|c|}
\hline \hline$i$ & $x_{i}^{*}$ & $i$ & $x_{i}^{*}$ & $i$ & $x_{i}^{*}$ & $i$ & $x_{i}^{*}$ \\
\hline 1 & $1.0035149867 \ldots$ & 3 & $1.0331118257 \ldots$ & 5 & $1.0443895684 \ldots$ & 7 & $1.0166082889 \ldots$ \\
2 & $1.0166082889 \ldots$ & 4 & $1.0443895684 \ldots$ & 6 & $1.0331118257 \ldots$ & 8 & $1.0035149867 \ldots$ \\
\hline \hline
\end{tabular}

Table 1: Numerical solution $\bar{x}^{*}$ of $(38)$

\begin{tabular}{rcccccc}
\hline \hline$\lambda$ & $\nu$ & $\mathcal{C}$ & $I$ & $T$ & $q_{I}$ & $\breve{\rho}$ \\
\hline 0 & 1 & 2909.34 & 5 & 311.997 & 3673 & 4.00725 \\
-1 & 0 & 2909.34 & 5 & 311.855 & 2697 & 4.00562 \\
-1 & 1 & 3166.88 & 5 & 332.769 & 2891 & 4.00604 \\
\hline \hline
\end{tabular}

Table 2: Numerical results for nonlinear system (38) with $m=8$

where $F=\left(F_{1}, \ldots, F_{m}\right)$.

Note that the last criterion is independent of the knowledge of the root (see $[8,10])$. Instead of the computational order of convergence defined in [21], we consider the parameter $P C L O C$ denoted by $\breve{\rho}$ and defined by (see [10])

$$
\breve{\rho}=\frac{\log \left\|F\left(x_{I}\right)\right\|}{\log \left\|F\left(x_{I-1}\right)\right\|} .
$$

On the other hand, the numerical results of this example are in Table 2, where we show the values of $\lambda$ and $\nu$ of the method, the cost $\mathcal{C}$, the necessary iteration number $I$, the elapsed time $T$ in milliseconds to reach iteration $I$, the number of correct decimals in $x_{I}$, denoted by $q_{I}$ and the parameter of local order of convergence $\breve{\rho}$.

If $\rho=\breve{\rho} \pm \Delta \breve{\rho}$, where $\rho$ is the local order of convergence and $\Delta \breve{\rho}$ is the error of PCLOC, we obtain $\Delta \breve{\rho}<10^{-3}$. This fact means that, in all computations of PCLOC, we obtain at least three significant digits, so that it is a good check of the local convergence orders of family (7).

The numerical computations have been performed on MPFR library of $\mathrm{C}++$ multiprecision arithmetics with 4096 digits of mantissa [7]. All programs have been compiled by g++(4.2.1) for i686-apple-darwin1 with libgmp (v.5.0.2) and libmpfr (v.3.1.0) libraries in a processor Intel@ Xeon E5620, 2.4GHz (64-bit machine). In this machine, the ratio $\ell$ is $\ell=1.731$ and note that, for each scalar function, we have 9 products and 1 quotient, so that $\mu=10.728$.

\section{Concluding remarks}

The family of fourth-order Steffensen-type methods proposed by Zheng, Li and Huang in [22] for solving scalar equations is optimal in the sense of the Kung-Traub conjecture [11]. In this work, we extend this family of iterative methods for solving systems of 
nonlinear equations and propose the most efficient. This extension uses multidimensional divided differences of first and second orders and every method of the family has local order of convergence at least four. We also analyse, from a certain computational efficiency index, the efficiency of every method of the family and identify two optimal methods. In addition, we study the semilocal convergence of one of these optimal methods by using a technique based on recurrence relations. Finally, we consider a numerical example where three of the most efficient methods of the family are applied to solve a system of nonlinear equations that arises from a process of discretization of a nonlinear integral equation of Hammerstein type. The numerical example confirms all the theoretical results presented in this work.

Acknowledgement: The authors would like to thank the anonymous reviewers for their valuable comments and suggestions to improve the quality of the paper.

\section{References}

[1] S. Amat, S. Busquier, A two-step Steffensen's method under modified convergence conditions. J. Math. Anal. Appl. 324, 1084-1092 (2006)

[2] S. Amat, S. Busquier, On a Steffensen's type method and its behavior for semismooth equations. Appl. Math. Comput. 177, 819-823 (2006)

[3] I.K. Argyros, The secant method and fixed points of nonlinear operators. Monatsh. Math. 106, 85-94 (1988)

[4] I.K. Argyros, On the secant method, Publ. Math. Debrecen 43, 223-238 (1993)

[5] I.K. Argyros, Computational Theory of Iterative Methods, in: C. K. Chui, L. Wuytack (Eds.), Series: Studies in Computational Mathematics, vol. 15. Elsevier, New York (2007)

[6] I.K. Argyros, H. Ren, Efficient Steffensen-type algorithms for solving nonlinear equations. Int. J. Comp. Math. 90, 691-704 (2013)

[7] L. Fousse, G. Hanrot, V. Lefèvre, P. Pélissier, P. Zimmermann, MPFR: A multiple-precision binary floating-point library with correct rounding. ACM Trans. Math. Softw. 33, 2, Art. 13 (15 pp) (2007)

[8] M. Grau-Sánchez, M. Noguera, J.M. Gutiérrez, On some computational orders of convergence. Appl. Math. Lett. 23, 472-478 (2010)

[9] M. Grau-Sánchez, A. Grau, M. Noguera, Frozen divided difference scheme for solving systems of nonlinear equations. J. Comput. Appl. Math. 235, 1739-1743 (2011)

[10] M. Grau-Sánchez, A. Grau, M. Noguera, J.R. Herrero, On new computational local orders of convergence. Appl. Math. Lett. 25, 2023-2030 (2012)

[11] H. T. Kung, J. F. Traub, Optimal order of one-point and multipoint iteration. Journal of the Association for Computing Machinery 21, 634-651 (1974)

[12] Z. Liu, Q. Zheng, P. Zhao, A variant of Steffensen's method of fourth-order convergence. Appl. Math. Comput. 216, 1978-1983 (2010)

[13] A. D. Polyanin, A. V. Manzhirov, Handbook of Integral Equations. CRC Press, Boca Ratón (1998)

[14] F.A. Potra, A characterisation of the divided differences of an operator which can be represented by Riemann integrals. Revu. Anal. Numer. Theor. Approx. 2, 251253 (1980)

[15] F.A. Potra, V. Pták, Nondiscrete Induction and Iterarive Processes. Pitman Publishing, Boston (1984) 
[16] H. Ren, Q. Wu, W. Bi, A class of two-step Steffensen type methods with fourth-order convergence. Appl. Math. Comput. 209, 206-210 (2009)

[17] J.R. Sharma, H. Arora, An efficient derivative free iterative method for solving systems of nonlinear equations. Appl. Anal. Discrete Math. 7, 390-403 (2013)

[18] J.R. Sharma, H. Arora, M.S. Petković, An efficient derivative free family of fourth order methods for solving systems of nonlinear equations. Appl. Math. Comput. 235, 383-393 (2014)

[19] A.Y. Taylor, D. Lay, Introduction to Functional Analysis, 2nd edition, New York, Wiley (1980)

[20] X. Wang, T. Zhang, A family of Steffensen type methods with seventh-order convergence. Numer. Algor. 62, 429-444 (2013)

[21] S. Weerakoon, T.G.I. Fernando, A variant of Newton's method with accelerated third-order convergence. Appl. Math. Lett. 13, 87-93 (2000)

[22] Q. Zheng, J. Li, F. Huang, An optimal Steffensen-type family for solving nonlinear equations. Appl. Math. Comput. 217, 9592-9597 (2011) 